\title{
Acute Encephalomyelitis during an Outbreak of Enterovirus Type 71 Infection in Taiwan: Report of an Autopsy Case with Pathologic, Immunofluorescence, and Molecular Studies
}

Chuen Hsueh, M.D., Shih-Ming Jung, M.D., Shin-Ru Shih, Ph.D., Tseng-tong Kuo, M.D., Ph.D., Wun-Ju Shieh, M.D., Ph.D., Sherif Zaki, M.D., Tzou-Yien Lin, M.D., Luan-Yin Chang, M.D., Hsiao-Chen Ning, M.S., Da-Chin Yen, M.D.

From the Department of Pathology, Chang Gung Memorial Hospital, Tao Yuan, Taiwan (CH, SMJ, TK); Chang Gung University, School of Medical Technology, Tao Yuan, Taiwan (SRS); Department of Pediatrics, Chang Gung Children's Hospital, Tao Yuan, Taiwan (TYL, LYC, DCY); and Infectious Disease Pathology Activity, Division of Viral and Rickettsial Diseases, Centers for Disease Control and Prevention, Atlanta, Georgia, USA (WJS, SZ)

We report a fatal case of enterovirus type 71 (EV 71) infection in an 8-year-old girl during a summer outbreak of hand, foot, and mouth disease in 1998 in Taiwan. The clinical course was rapidly progressive, with manifestations of hand, foot, and mouth disease, aseptic meningitis, encephalomyelitis, and pulmonary edema. The patient died 24 hours after admission. Postmortem study revealed extensive inflammation in the meninges and central nervous system and marked pulmonary edema with focal hemorrhage. Brain stem and spinal cord were most severely involved. The inflammatory infiltrates consisted largely of neutrophils involving primarily the gray matter with perivascular lymphocytic cuffing, and neuronophagia. The lungs and heart showed no evidence of inflammation. EV 71 was isolated from the fresh brain tissues and identified by immunofluorescence method with type-specific EV 71 monoclonal antibody. It was also confirmed by neutralization test and reverse-transcriptase polymerase chain reaction with sequence analysis. The present case was the first example in which EV 71 was demonstrated to be the causative agent of fatal encephalomyelitis during its epidemic in Taiwan.

Copyright $\odot 2000$ by The United States and Canadian Academy of Pathology, Inc.

VOL. 13, NO. 11, P. 1200, 2000 Printed in the U.S.A.

Date of acceptance: June 21, 2000.

Address reprint requests to: Tseng-tong Kuo, M.D., Ph.D., Department of Pathology, Chang Gung Memorial Hospital, 199 Tun Hwa North Road, Taipei 105, Taiwan; e-mail: ttkuo@adm.cgmh.com.tw; fax: +886-3-3280147.
KEY WORDS: encephalitis, encephalomyelitis, enterovirus, enterovirus type 71, hand, foot, and mouth disease, meningitis

Mod Pathol 2000;13(11):1200-1205

Enterovirus type 71 (EV 71), a member of the genus enterovirus within the family picornavirus, was initially isolated from the feces of a 9-month-old infant with fatal encephalitis in California in 1969 (1). Subsequent outbreaks were encountered in New York, Australia, Sweden, Japan, Bulgaria, Hungary, Hong Kong, China (2), and Malaysia (3). The epidemic seasons were in the summer and fall. Infants and young children were primarily affected. The clinical manifestations were noted to be variable, including hand, foot, and mouth disease (HFMD), aseptic meningitis, encephalitis, acute flaccid paralysis, and Guillain-Barré syndrome $(2,4,5)$. The predominating illness might be HFMD, central nervous system (CNS) disease, or both. In the summer of 1998, an outbreak of HFMD with more than 100,000 cases occurred in Taiwan. Most of the patients with severe CNS disease and high mortality were under the age of 5 years. Of the 78 patients who died, 65 of them had a rapid progression of pulmonary edema leading to death (5). Other than EV 71, Coxsackie virus A16 causes HFMD most frequently. The disease is usually self-limited without neurological deficits (6), whereas in HFMD associated with EV 71, serious and lethal complications may develop. There were two such outbreaks of EV 71 with rapid deterioration to death in young children previously $(3,7)$. One was in Bulgaria in 1975 and the other was in Malaysia in 1997. The Bulgarian outbreak drew global attention to EV 71 infection because of a high incidence of paralysis and 
mortality in young children. Of 705 patients, 149 developed paralysis and 44 died. The fatality rate among children was 93\%. Autopsy examination showed brain stem encephalitis in the majority of cases (7). However, pulmonary edema or hemorrhage was not reported in Bulgarian epidemic. In contrast, the frequency of pulmonary edema or hemorrhage was considerably high in the fatal cases in Taiwan and Malaysia (3-5). We report the first such case proved by postmortem studies in an 8-year-old girl during this outbreak. The clinicopathologic features and molecular studies are presented.

\section{CASE REPORT}

An 8-year-old girl was brought to our emergency room at 4:20 a.m. on June 7, 1998. She had been well until 2 days before, when she had developed a fever of $39.2^{\circ} \mathrm{C}$, oral ulcers, headache, and vomiting. Vesicular rashes were found on her hands and the soles of her feet 1 day later. On admission, physical examination revealed an alert girl with isocoric pupils and prompt light reflexes. The body temperature was $37.2^{\circ} \mathrm{C}$, heart rate was $114 / \mathrm{min}$, and blood pressure was 114/64 mm Hg. The throat was congested. Breathing sounds were clear and no cyanosis was found. Heartbeat was regular without gallop. No focal neurological signs were noted. Small papules were found on the soles of both feet. Her neck was stiff and her urinary bladder was distended. Laboratory studies revealed a white blood cell (white blood cell count) count of 14,300 cells/ $\mathrm{mm}^{3}$ and hemoglobin of $12.3 \mathrm{~g} / \mathrm{dL}$. Blood sugar was $134 \mathrm{mg} / \mathrm{dL}$ and the aspartate aminotransferase was $23 \mathrm{U} / \mathrm{L}$. The MB fraction of creatine kinase was 2 U/L. Cerebrospinal fluid analysis showed pleocytosis with 153 white blood cells $/ \mathrm{mm}^{3}$ and $70 \%$ neutrophils. The cerebrospinal fluid protein concentration was $43 \mathrm{mg} / \mathrm{dL}$ and the glucose was $76 \mathrm{mg} / \mathrm{dL}$. No organisms were seen on Gram-stained smear. Initial chest radiograph and brain computed tomography (CT) showed no abnormalities. Abdominal radiograph revealed dilated bowel loops and a distended urinary bladder. Throat and rectal swabs were taken for viral cultures. Intravenous penicillin and ceftizoxime were given. Initially, the patient complained of bilateral knee pain with itching and painful sensation of the right arm followed by severe headache and vomiting. No impairment of her muscle power was found at the time. Approximately 8 hours after admission, she became irritable and delirious with involuntary movement and self-talking. Tachypnea and tachycardia were noted also. Then she developed cyanosis and coughed up blood-tinged sputum. The patient was transferred to intensive care unit and was intubated and me- chanically ventilated. Pink fluid followed by fresh blood came out from the endotracheal tube. A chest radiograph revealed bilateral coarse pulmonary infiltrates. Coagulation profile revealed a prothrombin time of 15.3 seconds and a partial thromboplastin time of 46.9 seconds. Follow-up data showed white blood cell count of 31,800 cells $/ \mathrm{mm}^{3}$, blood sugar of $254 \mathrm{mg} / \mathrm{dL}$, and aspartate aminotransferase level of $68 \mathrm{U} / \mathrm{L}$. She became hypoxic with severe metabolic acidosis and rapid decline of her consciousness level. Bradycardia and hypotension developed despite cardiopulmonary resuscitation. The patient died 4 hours after intubation at 4:50 a.m. on June 8, 1998.

\section{MATERIALS AND METHODS}

\section{Histologic and Immunofluorescence Studies}

Autopsy was performed 12 hours postmortem. Tissues from various organs were fixed in $10 \%$ buffered formalin and embedded in paraffin. Fresh tissues from frontal and parietal lobes, pons, medulla, and cervical, thoracic, and lumbar spinal cords as well as heart, lung, liver, kidney, and pancreas were sampled for viral culture and further identification. Samples for virus isolation were inoculated into human embryonic fibroblast (MRC-5), RD cell, Hep-2, and LLC-MK2 cultures. The cultured cells with $50 \%$ cytopathic effect (CPE) were scraped and stained by immunofluorescence method. The monoclonal antibody used in the study was raised against EV 71 (Chemicon International, Inc., Temecula, CA, USA). The cells infected with EV 71 exhibited apple-green fluorescence in the nuclei and/or cytoplasm. Neutralization test was performed for confirmation (rabbit anti-EV71 antiserum against Japanese HFMD strain isolated in 1973 was kindly provided by Dr. H. Shimizu, NIH, Tokyo, Japan).

\section{Reverse Transcriptase-Polymerase Chain Reaction (RT-PCR) Amplification of EV 71 Genome}

EV 71-infected culture cells from the specimens of spinal cord and brain stem were lysed by $1 \mathrm{~mL}$ TRIzol reagent (Life Technologies, Grand Island, NY, USA) and followed by phenol-chloroform extraction and isopropranol precipitation. Eight sets of primers were used for amplification and sequencing of the entire genome of the viral DNA. They were designed from published sequence data of EV 71 BrCr strain: primer pair I, 5'-TTAAAACAGCTGTGGGTTG [nucleotide (nt) 1-19] as the sense strand primer, 5'-GAACTTCCAGTACCACCCC (nt 1196-1178) as the antisense strand primer; primer pair II, 5' -TATGGTGAGTGGCCTTCATACTG (nt 1053- 
75) as the sense strand primer, $5^{\prime}$-AGTGAGTGTTACTGATCCATGGT (nt 2241-2218) as the antisense strand primer; primer pair III, 5' - TACACACCACCAGGAGGCCCCT (nt 2115-2126) as the sense strand primer, 5'-ACCAGCATAATTTGGGTTGGCT (nt 32823261) as the antisense strand primer; primer pair IV, 5'-TTCCATTCATGT -CACCTGCGA (nt 2964-2984) as the sense strand primer, 5'-GAAATGGGCGAG -GTATGACACA (nt 4291-4269) as the antisense strand primer; primer pair V, 5'ATTAGTAAGTTTATTTATTGGCT(nt 4149-4171) as the sense strand primer, 5' -ACAACTGCAACCACAGTTGCTAT(nt 52715249) as the antisense strand primer; primer pair VI, 5'-TCCATTGCTACTGTGGTAGCAGT (nt 5253-5275) as the sense strand primer, $5^{\prime}$-TAGCAGGCTTCTTCCATTCTCAT (nt 6241-6219) as the antisense strand primer; primer pair VII, 5'-ACTAAGCTGGAACCCAGTG (nt 6003-6021) as the sense strand primer, 5' -ATAACAAATTTACCCCCACC (nt 7396-7377) as the antisense strand primer; primer pair VIII, 5'GAGAAATTTGTGAGTACAA (nt 7227-7245) as the sense strand primer, 5'-AGCAGTGGTAACAACGCAGAG-TACT(30)CN (nt 7408-7342). RT-PCR was carried out by RT-PCR beads (Pharmacia Biotech, Sweden). The reactions were placed in a Perkin Elmer Cetus DNA 480 thermal cycler programmed to incubate for $30 \mathrm{~min}$ at $42^{\circ} \mathrm{C}$ and $5 \mathrm{~min}$ at $94^{\circ} \mathrm{C}$, followed by 35 cycles of the following steps: denaturation at $94^{\circ} \mathrm{C}$ for $30 \mathrm{sec}$, annealing at $50^{\circ} \mathrm{C}$ for $30 \mathrm{sec}$, elongation at $72^{\circ} \mathrm{C}$ for $1.5 \mathrm{~min}$, and an additional $15 \mathrm{~min}$ for elongation in the last cycle. RT-PCR products were visualized on $1 \%$ agarose gel stained with ethidium bromide.

\section{Nucleotide Sequence of EV 71 Genome}

Nucleotide sequence was determined by an autosequencing system made by the program of the Genetic Computer Group (GCG) sequence analysis package.

\section{RESULTS}

\section{Gross Findings}

The heart revealed mild biventricular hypertrophy. Marked pulmonary congestion, edema, and focal hemorrhage were seen in both lungs. Mild focal subcapsular hemorrhage was found in the liver. Other organs were grossly unremarkable. The brain weighed $1350 \mathrm{~g}$. No evidence of herniation or edema was seen. No visible ischemic change was noted except that the gray matter of the spinal cord showed discoloration throughout.

\section{Histologic Findings}

Extensive inflammation was present in hypothalamus, fornix, gray matter of the frontal and parietal lobes of cerebrum, and dentate nuclei of the cerebellum as well as in brain stem and spinal cord. The degree and extent of inflammation were more severe in brain stem and spinal cord. The areas of involvement were inferior olivary nuclei, cranial nerve nuclei, and reticular formation of the medulla and pons, basis pontis, substantia nigra, and red nuclei of midbrain and gray matter of the cervical, thoracic, and lumbar spinal cords. The involved neurons showed degeneration and necrosis with neuronophagia (Fig. 1). The inflammatory infiltrates were primarily composed of neutrophils and microglial cells (Fig. 2). The inflammatory cells in leptomeninges were predominantly lymphocytes. In addition, perivascular cuffing with mononuclear cells was present (Fig. 3). No viral inclusion bodies were found. The lungs revealed primarily marked pulmonary edema with multifocal hemorrhage. Although no diffuse alveolar damage was present, the beginning of the hyaline membrane formation was noted in several areas. No inflammatory infiltrate or viral inclusion was found. The heart showed mild hypertrophy with a small focus of mononuclear cell infiltration in the myocardium of right ventricle. However, no accompanying myocyte damage or viral inclusion was seen. The liver showed mild microvesicular fatty change. The rest of the visceral organs were unremarkable.

\section{Ultrastructural Findings}

Ultrastructural examination revealed degeneration, vacuolar change, and membrane-bound vesicles within the mitochondria of the neurons in brain stem and spinal cord. No virus-like particles were found.

\section{Virus Isolation}

Virus was isolated from different CNS tissues, including frontal and parietal lobes of the brain,

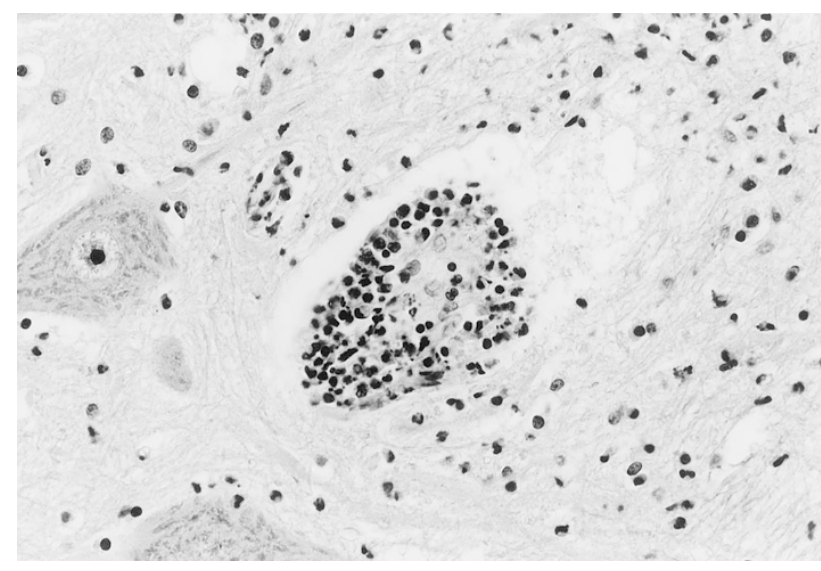

FIGURE 1. Aggregates of inflammatory cells with neuronophagia in the ventral horn of spinal cord [hematoxylin and eosin (H\&E) stain; original magnification, $100 \times]$. 


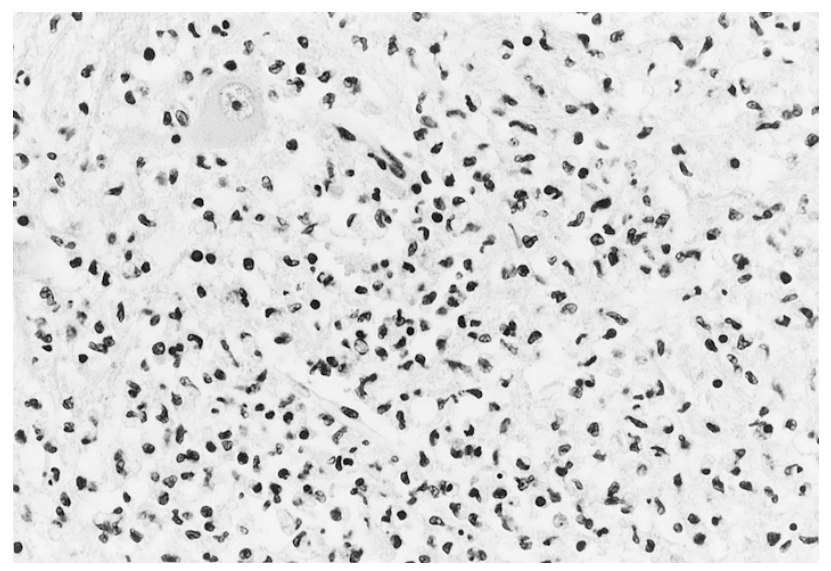

FIGURE 2. The inflammatory infiltrate consisted largely of neutrophils (H\&E stain; original magnification, $400 \times$ ).

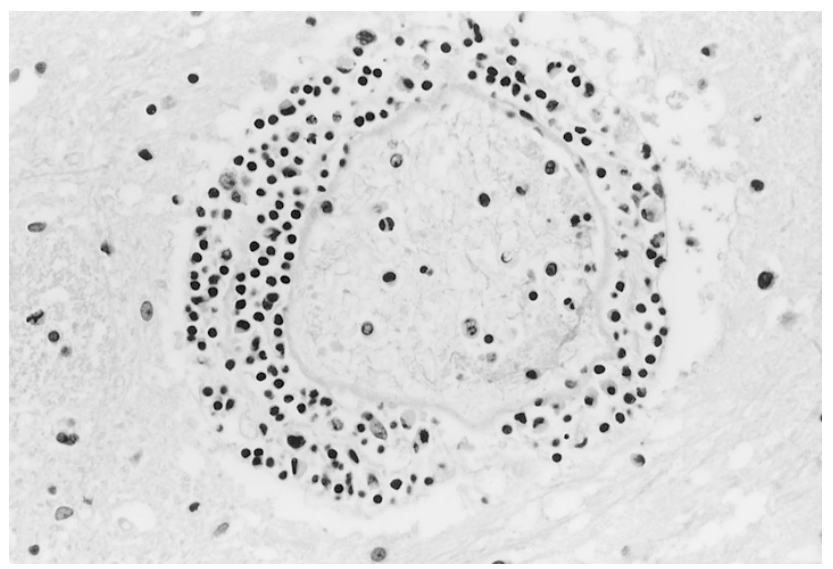

FIGURE 3. Perivascular lymphocytic cuffing in brain stem (H\&E stain; original magnification, $400 \times$ )

pons, medulla, cerebellum, and cervical, thoracic and lumbar spinal cord, as well as cerebrospinal fluid, whereas viral cultures from visceral organs, including heart, lungs, liver, kidney, and pancreas, revealed negative results. Isolates from spinal cord and brain tissue showed cytopathic effect characterized by shrinkage or elongation of cells with pyknotic nuclei. These viral isolates were stained with panenteroviral antibody and EV 71 monoclonal antibody (Chemicon International, Inc., Temecula, CA) and demonstrated apple green color in cytoplasm and nucleus under immunofluorescence microscope (Fig. 4).

\section{RT-PCR for the EV 71 Genome and Nucleotide Sequence}

The RT-PCR products with predicted size were obtained from RNA extract from spinal cord and the $5^{\prime}$-untranslated region of EV71 was detected by electrophoresis through $1 \%$ agarose gel (Fig. 5). The nucleotide sequence data has been deposited in the GenBank with the accession number AF119795 for entire genome isolated from spinal cord. The spec-

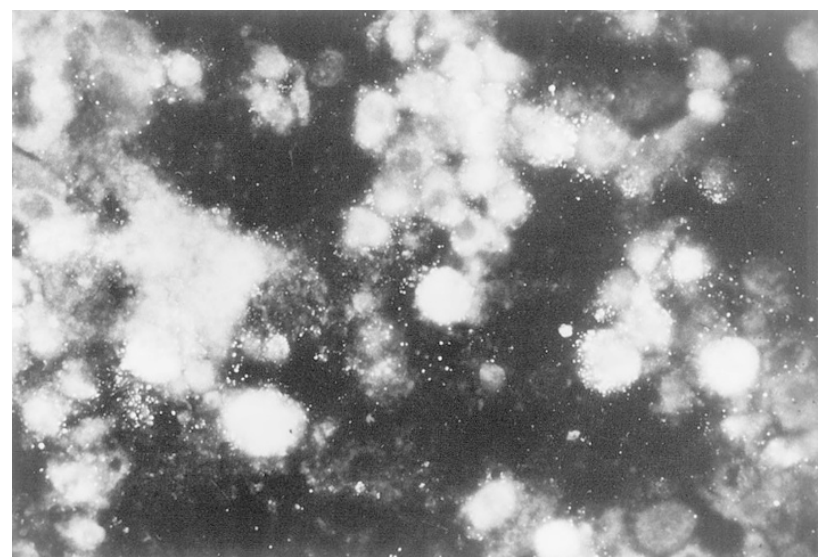

FIGURE 4. EV 71 viral antigen in the nucleus and the cytoplasm of infected cells was demonstrated by immunofluorescence study (original magnification, $400 \times)$.

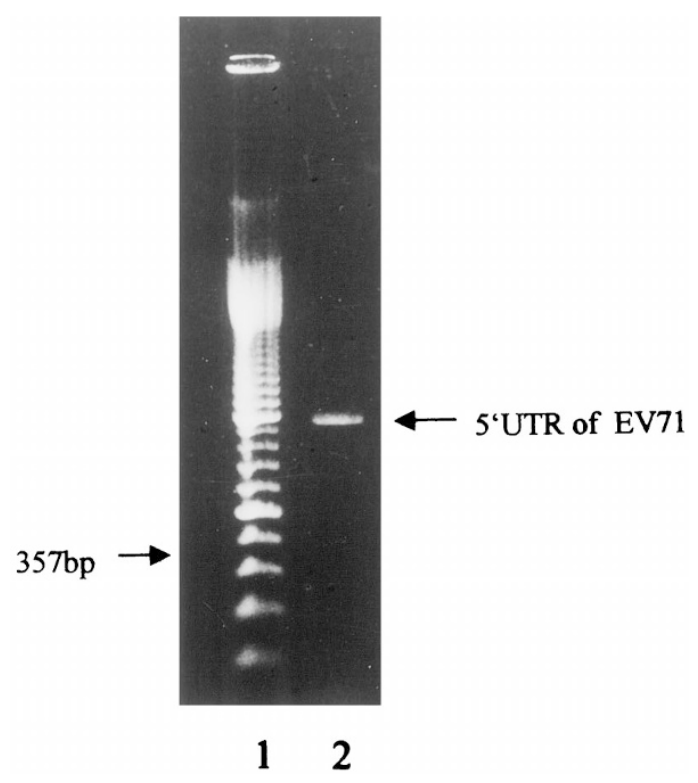

FIGURE 5. RT-PCR detection of 5'-untranslated region of EV71. The products were detected by electrophoresis through $1 \%$ agarose gel. The bands migrating at the predicted size were indicated. Lane 1 contained a 100-base-pair DNA ladder.

imen was also sent to the Centers for Disease Control (CDC) in the United States. The immunopositivity to anti-EV 71 antibody was demonstrated in the brain stem and spinal cord tissues and the isolate from the spinal cord tissue was confirmed to be EV 71 by RT-PCR. Picornavirus particles approximately $18 \mathrm{~nm}$ in diameter in the degenerated neurons of the spinal cord were found by electron microscopy at the CDC (8).

\section{DISCUSSION}

The topographic distribution of EV 71 infection in CNS is localized to brain stem, basal ganglia and spinal cord $(3,6,7,9)$. Our findings were comparable with those described in previous reports: de- 
spite widespread inflammation in CNS, the gray matter of spinal cord and brain stem was most severely involved, the areas from which EV 71 was isolated. The affected neurons were mostly destroyed and replaced by inflammatory cells. Because the ischemic change was not observed in unaffected neurons and hypoxia-susceptible regions, including Purkinje cell and neurons in the hippocampus and thalamus. The tissue damage in EV 71 encephalitis was more likely to have been caused by virus neurotropism than by ischemic injury, which has been postulated by some workers because of the paucity of inflammatory reaction in the lesions (10). The inflammatory infiltrate, consisting largely of neutrophils in our case, seemed to be unusual for viral infection in general. However, such observations had been described in previous reports of EV 71 encephalitis (3). In fact, the presence of dense neutrophilic infiltration is not uncommon in the early stages of some forms of acute viral encephalitis (11).

The fulminant clinical course with meningitis, brain stem encephalitis, pulmonary edema, and rapid progression to death in our patient was typically seen in the fatal cases in this particular outbreak of $\operatorname{EV} 71(4,5,12)$. Although the causative agent was EV 71, the major contributing factor to such a rapid deterioration to death was thought to be pulmonary edema, which was most probably noncardiogenic. Because myocarditis can be one of the lethal complications in EV 71 infection, it should always be excluded in fatal cases. We could find only a small focus of mononuclear cell infiltration without accompanying myocyte damage in myocardium on postmortem examination. It was not sufficient to render the diagnosis of myocarditis. In addition, no clinical manifestations suggestive of myocarditis were present nor was EV 71 isolated from the heart. Other causes of noncardiogenic pulmonary edema, such as overwhelming viremia, pneumonia, and diffuse alveolar damage, were unlikely, because there was no substantial evidence to support these diagnoses based on clinical and pathological grounds. Although the beginning of hyaline membrane formation was seen in the lungs, the process was mild and focal, not to the extent of diffuse alveolar damage.

The pulmonary edema associated with brain stem encephalitis has been proposed to be neurogenic, caused by the EV 71 involvement of the vasomotor and respiratory centers in the lower brain stem $(3,13)$. The extensive involvement of reticular formation in brainstem and gray matter of spinal cord could explain the development of neurogenic pulmonary edema $(4,5,12)$. Other than that, the presence of neurogenic bladder, paralytic ileus, and hyperglycemia could also be explained by the destruction of autonomic system in brainstem and spinal cord. In the early phase of brain stem encephalitis, overt neurologic signs and disturbance of consciousness are usually absent, as it was in our case. Although not seen in the present case, acute flaccid paralysis is one of the CNS complications associated with EV 71 infection. It can be clinically indistinguishable from paralytic poliomyelitis at times because the patterns of spinal involvement are similar (14). Hence, EV 71 should be included in the differential diagnosis of paralytic disease and brainstem encephalitis in children.

There had been two previous outbreaks of EV 71 infection in Taiwan in 1980 and 1986, respectively (5). The epidemic areas were much smaller, the number of patients affected was fewer, and no fatality was reported. The variation in clinical presentations in different epidemics or in different patients in a particular epidemic might be caused by varying dermatotropic or neurotropic character of the virus strain involved (6). It seems that such difference depends upon the interaction between the binding sites of the virus and cell receptors of the target organs. Related studies have been primarily focused on poliovirus; single-base changes have been linked to its neurovirulence (9). Similar molecular investigations on EV 71 are not yet available. In the present case, the entire nucleotide sequence showed a $91 \%$ identity in all the coding regions compared with the published prototypes of BrCr strain in China (15). An overall 97\% nucleotide homology without mutations was detected compared with the BrCr strain in nonfatal cases in the same outbreak, and a $90 \%$ nucleotide homology was found compared with cases in two previous outbreaks in Taiwan (S.-R. Shih, unpublished data). However, the virus strains in Taiwan were found to be genetically distinct from the strains in Malaysia (16). The molecular genetics of EV 71 strains involved in the epidemic are still under investigation in Taiwan. Questions regarding the change of viral antigenicity in relation to epidemicity of the disease as well as the significance of viral mutation and its correlation to clinical manifestations remain to be answered, yet no definite conclusions can be made now. It is important to recognize that EV 71 infection may cause rapid clinical deterioration to death in young children. Especially in cases such as ours, rapid progression to death was preceded by the development of brain stem encephalitis and pulmonary edema.

\section{REFERENCES}

1. Schmidt NJ, Lennette EH, Ho HH. An apparently new enterovirus isolated from patients with disease of the central nervous system. J Infect Dis 1974;129:304-9.

2. Melnick JL. Enteroviruses: polioviruses, coxsackieviruses, echoviruses, and new enteroviruses. In: Fields BN, Knipe 
DM, Howley PM, editors. Fields virology, 3rd ed. Philadelphia: Lippincott Raven; 1996. p. 655-712.

3. Lum LCS, Wong KT, Lam SK, Chua KB, Gob AYT, Lin WL, et al. Fatal enterovirus 71 encephalomyelitis. J Pediatr 1998; 133:795-8.

4. Huang CC, Liu CC, Chang YC, Chen CY, Wang ST, Yeh TF. Neurologic complications in children with enterovirus 71 infection. N Engl J Med 1999;341:936-42.

5. Ho M, Chen ER, Hsu KH, Twu SJ, Chen KT, Tsai SF, et al. An epidemic of entervirus 71 infection in Taiwan. N Engl J Med 1999;341:929-35.

6. Ishimaru Y, Nakano S, Yamaoka K, Takami S. Outbreak of hand, foot, and mouth disease by enterovirus 71: high incidence of complication disorders of central nervous system. Arch Dis Child 1980;55:583-8.

7. Chumakov M, Voroshilova M, Shindarov L, Larvrova I, Gracheva L, Koroliva G, et al. Enterovirus 71 isolated from cases of epidemic poliomyelitis-like disease in Bulgaria. Arch Virol 1979;60:329-40.

8. Goldsmith CS, Shieh WJ, Pallansch MA, Ksiazek TG, Hsueh C, Jung SM, et al. Fatal encephalitis in young children, Taiwan, 1998. Microsc Anal 1999;5:1146-7.
9. Rotbart HA. Enteroviral infections of the central nervous system. Clin Infect Dis 1995;20:971-81.

10. Landry ML, Fonseca SNS, Cohen S, Bogue CW. Fatal enterovirus type 71 infection: rapid detection and diagnostic pitfalls. Pediatr Infect Dis 1995;14:1095-101.

11. Esiri MM, Kennedy PG. Viral diseases. In: Greenfield's Neuropathology, $6^{\text {th }}$ ed Vol 12. Graham DI, Lantos P, editors. London:Arnold, 1997:11.

12. Chang LY, Huang YC, Lin TY. Fulminant neurogenic pulmonary oedema with hand, foot, and mouth disease. Lancet 1998;352:367-8.

13. Baker AB. Poliomyelitis: a study of pulmonary edema. Neurology 1957;7:745-51.

14. Melnick JL. Enterovirus type 71 infections: a varied clinical pattern sometimes mimicking paralytic poliomyelitis. Rev Infect Dis 1984;6(Suppl 2):387-90.

15. Zheng ZM, Zhang JH, Zhu WP, He PJ. Isolation of enterovirus type 71 from the vesicle fluid of an adult patient with handfoot-mouth disease in China. Virol Sinica 1989;4:375-82.

16. Deaths among children during an outbreak of hand, foot, and mouth disease -Taiwan, Republic of China. MMWR Morb Mortal Wkly Rep 1998;47:629-32.

\section{Book Review}

\section{Schumacher HR, Rock, WA Jr., Stass SA: Hand- book of Hematologic Pathology, 774 pp, New York, Marcel Dekker, Inc., 2000 (\$225)}

In the preface of this book the editors state that it "... was written by authors who apply every day what they write about in their chapters." Indeed, it was a pleasure to read this text because this "first-hand" experience can be felt throughout the book.

The particular strength of this book is the nonneoplastic hematopathology. Complex and difficult concepts are clearly presented, and tables are excellent throughout the book. Diagrams are very good. Case studies at the end of chapters are well chosen and accompanied by overall excellent discussions. The book is also problem-oriented, but this again applies mainly to non-neoplastic hema- tology. A short but excellent chapter on quality control and quality assurance in hematology laboratory is given at the end of the book.

The choice of figures is very good, but many figures are out of focus, the contrast is poor, and most are too dark. Some of the figures are so bad that they look like poor quality photocopies. I found one mislabeled figure (p. 178).

I would strongly recommend this book to residents and fellows in hematopathology. But make sure you also have a good hematopathology color atlas while studying for certification in hematopathology.

\section{Emina Torlakovic \\ The Norwegian Radium Hospital \\ Oslo, Norway}

